\title{
A 2-year randomised placebo-controlled trial of doxycycline for lymphangioleiomyomatosis
}

\author{
William Y.C. Chang ${ }^{1}$, Jennifer L. Cane ${ }^{1}$, Maruti Kumaran², Sarah Lewis ${ }^{3}$, \\ Anne E. Tattersfield ${ }^{1}$ and Simon R. Johnson ${ }^{1,4}$
}

Affiliations: ${ }^{1}$ Division of Therapeutics and Molecular Medicine, University of Nottingham, Nottingham, ${ }^{2}$ Dept of Radiology, Nottingham University Hospitals NHS Trust, Nottingham, ${ }^{3}$ Division of Epidemiology and Public Health, University of Nottingham, Nottingham, and "National Centre for Lymphangioleiomyomatosis, Nottingham University Hospitals NHS Trust, Nottingham, UK.

Correspondence: S.R. Johnson, Division of Therapeutics and Molecular Medicine, University of Nottingham, Nottingham, UK. E-mail: simon.johnsonanottingham.ac.uk

ABSTRACT Lymphangioleiomyomatosis (LAM) is characterised by lung cysts and airflow obstruction. Matrix metalloproteinases have been implicated in lung destruction in LAM. We performed a randomised, double-blind trial, comparing the matrix metalloproteinases inhibitor doxycycline with placebo on the progression of LAM.

23 females with LAM were randomised to doxycycline $100 \mathrm{mg}$ daily for 3 months followed by $200 \mathrm{mg}$ daily for 21 months, or matched placebo. Lung function, exercise capacity, quality of life and matrix metalloproteinases levels were measured.

21 patients completed 6 months of treatment, 17 completed 1 year of treatment and 15 completed 2 years of treatment. Eight withdrew from the trial due, four due to a pneumothorax and four because of other reasons. The mean \pm SD decline in FEV1, the primary endpoint, did not differ between the groups being $-90 \pm 154 \mathrm{~mL} \cdot$ year $^{-1}$ in the placebo group and $-123 \pm 246 \mathrm{~mL} \cdot$ year $^{-1}$ in the doxycycline group (difference $-32.5,95 \%$ CI $-213-148 ; p=0.35$ ). Doxycycline had no effect upon vital capacity, gas transfer, shuttle walk distance or quality of life. Urine matrix metalloproteinases- 9 measurements were lower with doxycycline treatment $(\mathrm{p}=0.03)$.

Although with limited numbers we cannot completely exclude an effect of doxycycline, the lack of effect on any outcome makes it unlikely that doxycycline has a useful effect in LAM.

@ERSpublications

Doxycycline does not prevent progression of lung disease in LAM http://ow.ly/tiMqo

For editorial comments see page 967.

This article has supplementary material available from www.erj.ersjournals.com

Received: Sept 252013 | Accepted after revision: Nov 012013 | First published online: Dec 052013

Clinical trial: This study is registered at www.clinicaltrialsregister.eu/ with the identifier number EUDRACT 2007-003745-32 Support statement: The British Lung Foundation (grant P07-2), LAM Action and the Jarron Family.

Conflict of interest: Disclosures can be found alongside the online version of this article at www.erj.ersjournals.com Copyright @ERS 2014 


\section{Introduction}

Lymphangioleiomyomatosis (LAM), a rare disease of the lungs and lymphatic system, which occurs almost exclusively in females, can occur as a sporadic disease or in patients with tuberous sclerosis complex (TSC). Pulmonary symptoms generally dominate the clinical picture as lung cysts form, causing a pneumothorax, airflow obstruction and progressive respiratory impairment [1]. The rate of disease progression varies considerably between patients, with the decline in forced expiratory volume in $1 \mathrm{~s}$ (FEV1) usually being between 70 and $140 \mathrm{~mL} \cdot$ year $^{-1}$ [2-4]. Lymphatic obstruction can lead to chylous pleural effusions, ascites and abdominopelvic masses and approximately half of the patients have renal angiomyolipomas, a benign mesenchymal tumour [1].

In patients with LAM the lungs and lymphatic system are infiltrated by LAM cells, a clonal cell population with bi-allelic inactivation of the TSC-2 gene leading to constitutive activation of mammalian target of rapamycin (mTOR) [5]. Targeting mTOR with sirolimus in patients with LAM has been shown to reduce the decline in FEV1 and angiomyolipoma volume [4, 6, 7].

LAM cells produce proteolytic enzymes, which may contribute to lung cyst formation [8, 9]. Matrix metalloproteinases (MMPs), can degrade extracellular matrix, and can affect cell growth, invasion, angiogenesis and inflammation [10]. MMP-2 and -9 are overexpressed in the serum of females with LAM $[11,12]$ and MMP-1, $-2,-9$ and -14 are strongly expressed in the lungs of patients, particularly adjacent to cysts where disrupted collagen and elastic fibres are observed [13, 14]. Therefore, inhibition of MMP activity could reduce lung destruction in LAM.

Doxycycline, a tetracycline antibiotic, inhibits the production and activity of several MMPs including MMP-1, -2 and -9, and it has reduced pathological tissue remodelling in models of vascular disease and tumour growth $[15,16]$. Doxycycline is the only MMP inhibitor licensed for clinical use and Moses et al. [17] described a large improvement in spirometry and oxygenation in a patient with advanced LAM following treatment with doxycycline. In an observational study of patients with mild LAM, doxycycline was associated with a reduction in urine MMP-9 and a relatively slow decline in mean FEV1 of 70 mL over 12 months [18, 19]. As a consequence of these reports, some females with LAM have been taking doxycycline off label. In order to determine if doxycycline could inhibit MMP activity and reduce lung destruction in LAM, we conducted a randomised study of doxycycline and placebo over a 2-year period, using rate of decline in $\mathrm{FEV}_{1}(\Delta \mathrm{FEV} 1)$ as the primary outcome measure. Other physiological measures, quality of life, MMP activity, safety and tolerability were also recorded.

\section{Methods}

\section{Study protocol}

Patients were eligible for the study if they were aged $>18$ years and had sporadic or TSC associated LAM, classified as "definite" by the European Respiratory Society criteria [20], and an <FEV1 80\% predicted or evidence of a $20 \%$ deterioration in FEV1. Patients were excluded if they were post lung transplant or if they had used mTOR inhibitors or had previously been diagnosed with a pneumothorax, chylous effusion or bleeding angiomyolipoma within the previous 3 months. Hormone and bronchodilator treatment for LAM was allowed, provided that treatment had not changed in the 3 months prior to enrolment. The study was approved by the Trent Multicentre Research Ethics committee (NRES 07/H0403/165) the Medicines and Healthcare Regulatory Agency (MHRA 03057/0032/001-002) and registered with the EU Clinical Trials Registry. All patients provided informed consent.

Patients had a medical history and physical examination and completed a St George's Respiratory Questionnaire (SGRQ) at baseline. Pulmonary function tests including postbronchodilator spirometry, gas transfer, lung volumes and endurance shuttle walk test were measured according to the Association for Respiratory Technology and Physiology/British Thoracic Society standards in a single laboratory [21]. Blood was drawn for haematology, biochemistry, liver function, C-reactive protein, and both blood and urine were taken for MMPs and other biomarkers analyses.

Patients were randomised to receive either doxycycline $100 \mathrm{mg}$ daily or matched placebo as a single tablet. After 3 months the dose was increased to two tablets of the active drug (200 mg doxycycline) or placebo. Patients were assessed every 3 months over a 2-year period. At 12 and 24 months, patients had a full evaluation as at baseline (fig. 1). Computed tomography (CT) scans of the thorax and abdomen were carried out at 0 and 24 months in patients giving additional consent. The longest dimension of the largest renal angiomyolipoma was measured by a radiologist (M. Kumaran) as previously described [7]. Patients were withdrawn from the study if there was a fall in FEV1 from the study baseline of $>300 \mathrm{~mL}$ on two consecutive visits or if there was a severe adverse event. Those experiencing a pneumothorax were also withdrawn from the study, as spirometry can take many months to return to baseline following pneumothorax (unpublished data). The full protocol is available in the online supplementary material. 


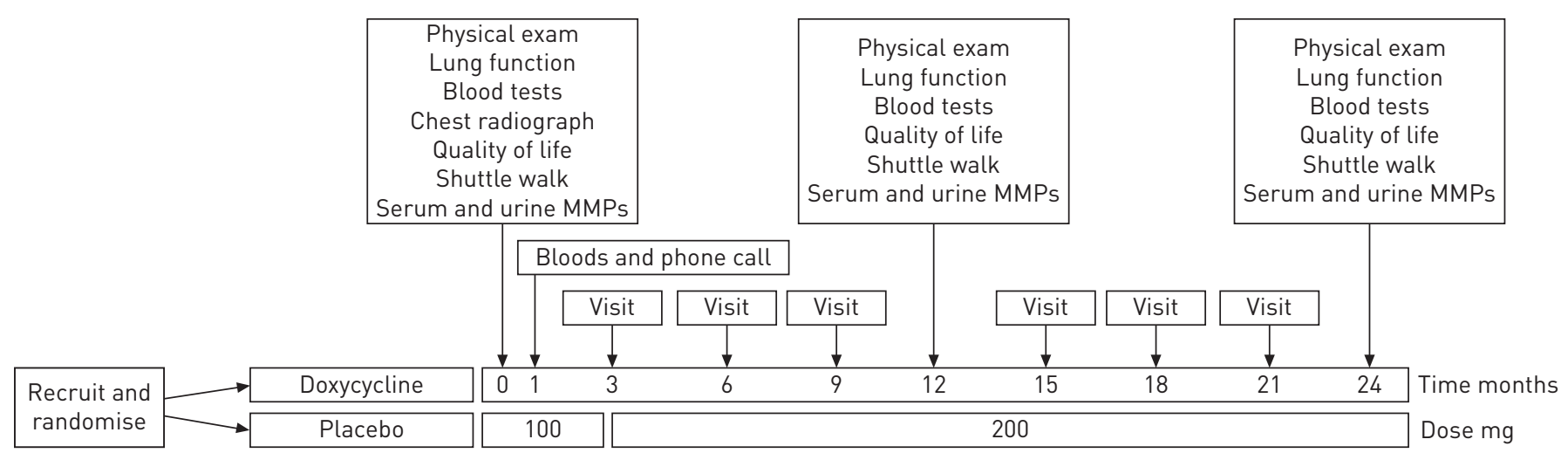

FIGURE 1 Outline of study protocol. Visit includes history, physical examination, post-bronchodilator spirometry and safety blood tests. MMPs: matrix metalloproteinases.

\section{MMP and vascular endothelial growth factor D measurements}

Serum total MMPs and vascular endothelial growth factor (VEGF)-D were measured using a Quantikine MMP-2 Immunoassay, Duoset Human MMP-9 Immunoassay and Human VEGF-D ELISA, respectively (all R\&D Systems, Minneapolis, MN, USA). Since MMPs are secreted as inactive zymogens, which require proteolytic cleavage for activation, we measured pro- and activated MMP-9 in serum and urine using gelatin zymography as previously described $[12,22]$.

\section{Analyses}

The primary outcome was rate of decline in postbronchodilator FEV1 over the course of the study analysed on an intention-to-treat basis for all patients. $\triangle \mathrm{FEV} 1$ was calculated by fitting a regression line to all postbronchodilator FEV1 measurements for each patient with the slope of this line expressed in $\mathrm{mL} \cdot \mathrm{year}^{-1}$. The effect of loss to follow-up was examined by comparing $\triangle \mathrm{FEV} 1$ between groups who only completed 6, 12, 18 and 24 months of treatment. For all endpoints normality was assessed by Kolmogorov-Smirnov statistic and mean values for the two groups were compared by two sample t-tests. Analyses were performed in Graphpad Prism version 5.00, (GraphPad Software, San Diego, CA, USA). Serum and urine MMP values were compared as log transformed values over time between treatment groups using a linear, mixed model, with repeated measures from individuals as a random effect in Stata 11 (Timberlake Consultants, London, UK).

\section{Results}

\section{Patient recruitment and baseline characteristics}

Patients were recruited over a 2-year period, starting in May 2009. After contacting 149 patients with LAM in the UK, 30 of those responding appeared to be suitable and were screened, of whom 23 were eligible. 12 patients were assigned to take doxycycline and 11 to take matched placebo (fig. 2). At recruitment the mean age of patients was 46 years and symptoms had been present for an average of 13.5 years. 18 (78\%) patients had been diagnosed in the past with a pneumothorax and $13(56 \%)$ had been diagnosed with an angiomyolipoma. A third of patents were post-menopausal. One patient had TSC and the remainder sporadic LAM. 21 out of the enrolled 23 patients had a serum VEGF-D level $>800 \mathrm{pg} \cdot \mathrm{mL}^{-1}$, which is considered the diagnostic level for LAM. [23] Patients had moderate-to-severe airflow obstruction with a mean FEV1 of 1.691 (58\% pred) and moderately impaired gas transfer of $4.38 \mathrm{kPa} \cdot \mathrm{min}^{-1} \cdot \mathrm{mL}^{-1}(51 \%$ pred). Baseline characteristics within the two groups were similar in terms of age, disease duration, clinical manifestations, menopausal status, quality of life and serum VEGF-D but mean FEV1 and transfer factor of the lung for carbon monoxide (TLCO) were slightly lower in the doxycycline group (tables 1 and E1).

\section{Effect of doxycycline on rate of decline of FEV1}

$\triangle \mathrm{FEV} 1$ analysed on an intention-to-treat basis expressed as mean $\pm \mathrm{SD}$ was $-90.3 \pm 154 \mathrm{~mL} \cdot \mathrm{year}^{-1}$ in the placebo group and $-123 \pm 246$ in the doxycycline group; the difference, $-32.5 \mathrm{~mL} \cdot \mathrm{year}^{-1}$ (95\% confidence interval -213-147.8), was not significant $(\mathrm{p}=0.35)$ (fig. 3). Patients not completing the study had a greater decline in FEV1 (fig. 4). $\triangle \mathrm{FEV} 1$ was $-36.3 \pm 63 \mathrm{~mL} \cdot \mathrm{year}^{-1}$ for all patients completing the study and $-240 \pm 302$ for those stopping early for any reason $(\mathrm{p}=0.049)$ : median values were $-30 \mathrm{~mL} \cdot \mathrm{year}^{-1}$ and $-162 \mathrm{~mL} \cdot$ year $^{-1}$, respectively. A sensitivity analysis showed there was no difference between doxycycline and placebo for any duration of treatment for the primary endpoint (table 2). 


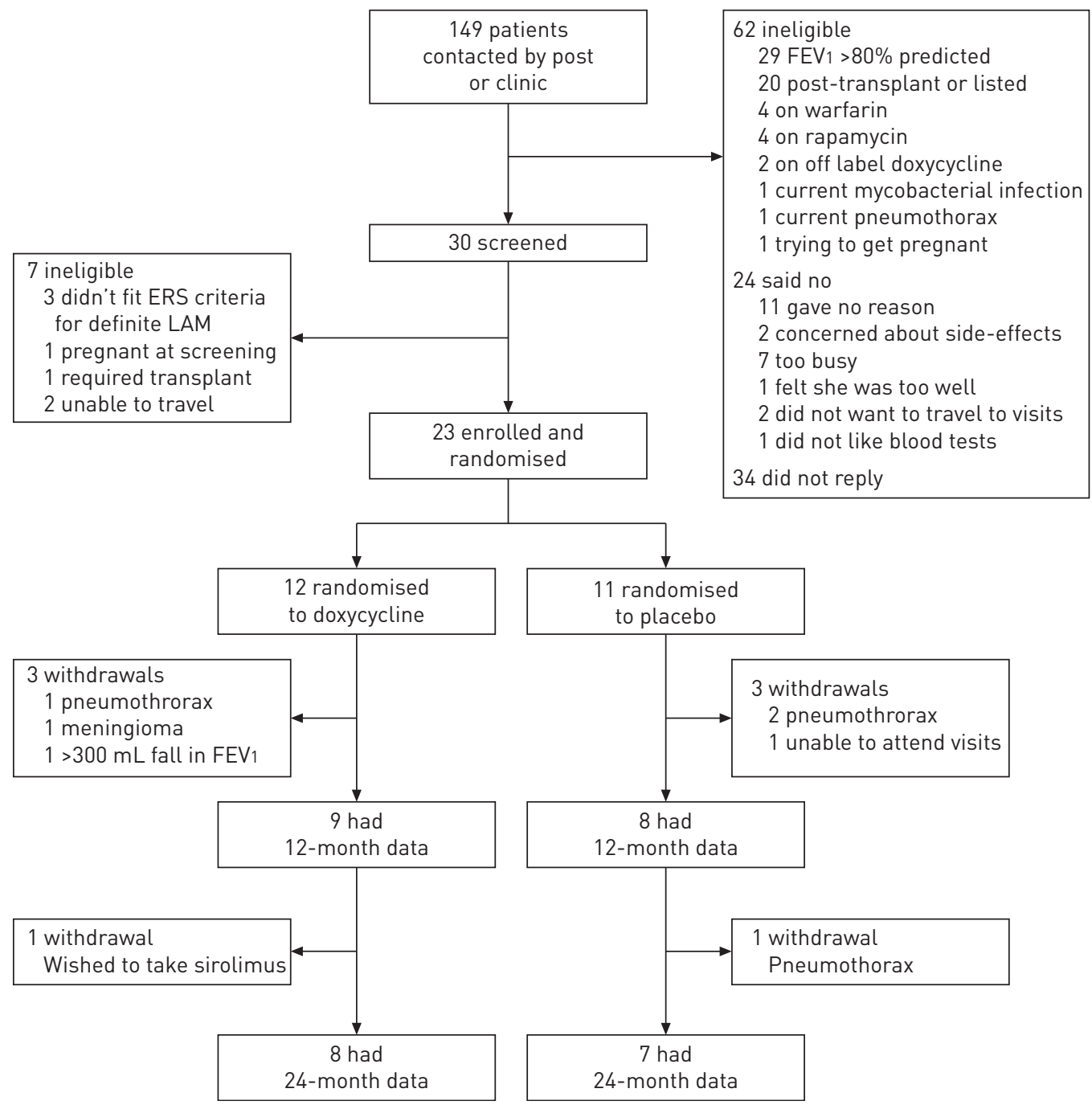

FIGURE 2 Recruitment and retention of participants. FEV1: forced expiratory volume in $1 \mathrm{~s}$; ERS: European Respiratory Society; LAM: lymphangioleiomyomatosis.

\section{Secondary endpoints}

Patients treated with doxycycline showed a $150 \mathrm{~mL}$ rise in forced vital capacity (FVC) after 12 months compared with placebo treatment, although no difference was present at 24 months. Otherwise, there were no differences between change in TLCO, shuttle walk distance or in quality of life scores between the two groups after 12 or 24 months of treatment (table 3). VEGF-D did not change significantly in either group over the course of the study (table 3). 20 patients underwent CT scanning of the chest and abdomen at baseline and 13 had a further scan at the end of the study. No LAM related complications, including chylous collections, developed in any patient during the study. A follow-up renal scan in six patients receiving placebo and six patients receiving doxycycline showed no change in the mean angiomyolipoma size in either group (fig. E1).

\section{Adverse events}

All patients were questioned for adverse events. Six patients were withdrawn because of adverse events. Of these six four patients this had a pneumothorax, (one doxycycline, three placebo), one patient had a fall in FEV1 $>300 \mathrm{~mL}$ (doxycycline) and one patient (doxycycline) had an epileptic seizure and was found to have a meningioma (fig. 2). Three patients in the placebo group and three in the doxycycline group had at least one respiratory infection requiring antibiotic treatment over the study period, with two patients in the doxycycline group having several respiratory infections (table 4). Although more adverse events were reported with doxycycline, only dyspepsia and photosensitivity were attributed to the drug. No significant disturbances in haematological or biochemical values occurred in either group. 
TABLE 1 Baseline characteristics of study subjects

\begin{tabular}{|c|c|c|c|}
\hline & All patients & Doxycycline & Placebo \\
\hline Subjects & 23 & 12 & 11 \\
\hline \multicolumn{4}{|l|}{ Patient characteristics } \\
\hline Age years & $46.5 \pm 9.0$ & $47.0 \pm 9.3$ & $45.7 \pm 8.9$ \\
\hline Duration of disease year & $13.5 \pm 9.1$ & $14.5 \pm 9.0$ & $12.5 \pm 9.4$ \\
\hline Post-menopause & $\overline{8}$ & $\overline{4}$ & $\overline{4}$ \\
\hline Pneumothorax $\#$ & 18 & 9 & 9 \\
\hline Angiomyolipoma & 14 & 6 & 8 \\
\hline TSC-LAM & 1 & 0 & 1 \\
\hline Supplemental oxygen use & 5 & 3 & 2 \\
\hline \multicolumn{4}{|l|}{ Lung function $\%$ predicted } \\
\hline FEV 1 & $58 \pm 23$ & $52 \pm 25$ & $64 \pm 19$ \\
\hline FVC & $95 \pm 22$ & $97 \pm 26$ & $92 \pm 18$ \\
\hline TLCO & $51 \pm 21$ & $45 \pm 21$ & $57 \pm 20$ \\
\hline TLC & $94 \pm 20$ & $99 \pm 23$ & $89 \pm 15$ \\
\hline \multicolumn{4}{|l|}{ Other parameters } \\
\hline Shuttle walk distance m & $573 \pm 286$ & $560 \pm 284$ & $586 \pm 301$ \\
\hline SGRQ total score & $35 \pm 4$ & $36 \pm 6$ & $33 \pm 6$ \\
\hline VEGF-D pg $\cdot \mathrm{mL}^{-1}$ & $2540 \pm 1377$ & $2347 \pm 1399$ & $2751 \pm 1387$ \\
\hline
\end{tabular}

Data are presented as $\mathrm{n}$ or mean $\pm \mathrm{SD}$. TSC: tuberous sclerosis complex; LAM: lymphangioleiomyomatosis; FEV1: forced expiratory volume in $1 \mathrm{~s}$; FVC: forced vital capacity; TLCO: transfer factor of the lung for carbon monoxide; TLC: total lung capacity; SGRQ: St George's Respiratory Questionnaire; VEGF-D: vascular endothelial growth factor D. \#: number of patients having had at least one pneumothorax. ": number of patients with one or more angiomyolipoma at any time.

\section{Effect of doxycycline on serum and urine MMPs}

At baseline MMP-2 and MMP-9 were present in serum on gelatin zymography (fig. 5), and mean \pm SD total serum for MMP-2 and MMP-9 levels, measured by ELISA, were $259 \pm 48 \mathrm{ng} \cdot \mathrm{mL}^{-1}$ and $265 \pm 173 \mathrm{ng} \cdot \mathrm{mL}^{-1}$, respectively. In urine, MMP-9 dimers, neutrophil gelatinase-associated lipocalin bound MMP-9, proMMP-9 and active MMP-9 were found, but not MMP-2 (fig. 5).

There was no significant difference in serum MMP-2 or -9 between groups over the 2-year period (fig. E3). Urine total and active MMP-9 values varied markedly between subjects at baseline and within subjects during the study in patients receiving placebo. This variation did not relate to infections as assessed clinically or by C-reactive protein and neutrophil counts (figs 5 and E4). There was a significant reduction in total urinary MMP-9 $(\mathrm{p}=0.03)$ and a reduction of borderline significance in active MMP-9 $(\mathrm{p}=0.07)$ in the doxycycline group when compared with placebo values over the 2-year period (fig. 5).
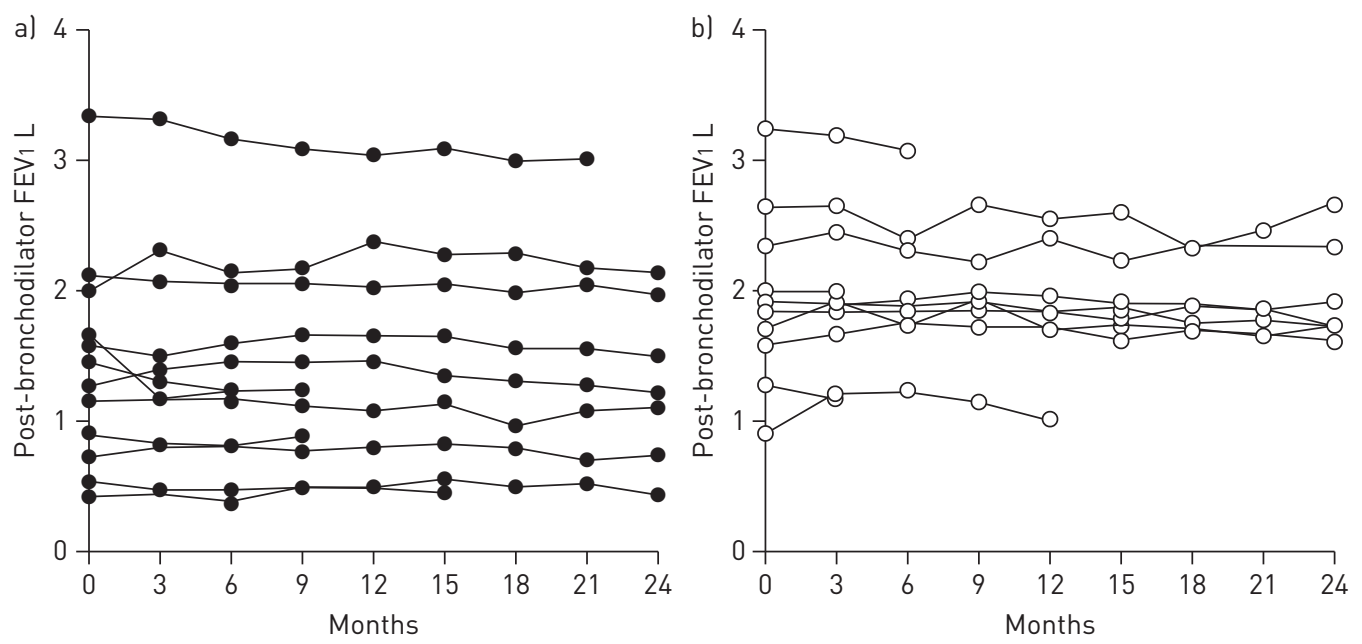

FIGURE 3 Serial post-bronchodilator forced expiratory volume in $1 \mathrm{~s}$ (FEV1) for all study participants. a) Doxycycline and b) placebo. 


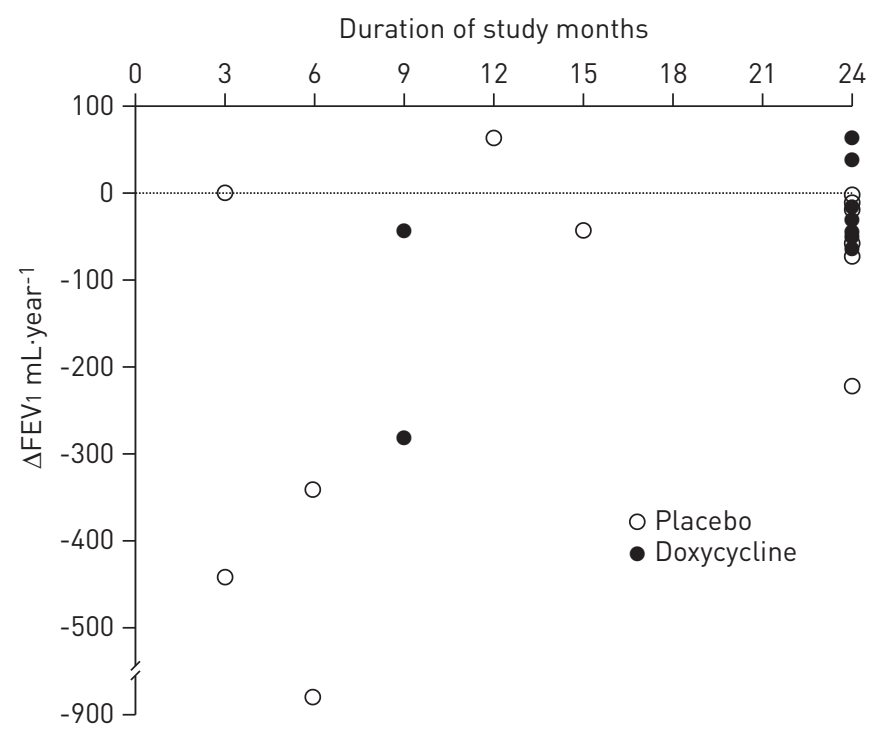

FIGURE 4 Rate of decline in forced expiratory volume in $1 \mathrm{~s}(\triangle \mathrm{FEV} 1)$ for individual participants compared with the time of study withdrawal. Patients with more rapid falls in FEV1 were more likely to withdraw from the study.

\section{Discussion}

We have conducted the first randomised, placebo controlled trial of doxycycline as a potential therapy for LAM. Doxycycline had no effect on the rate of decline of FEV1 over a 2-year period, and no effect on FVC, TLCO, total lung capacity, shuttle-walk distance or quality of life scores after 24 months of treatment. Because LAM is rare, the number of patients studied was relatively small. Our findings, therefore, cannot completely exclude an effect of doxycycline, although the lack of a sustained effect on any outcome makes it very unlikely that doxycycline has a useful effect that we have missed.

Study subjects were drawn from a national database and a clinical referral service that has evaluated over half the patients known to have LAM in the UK. Patients were similar to those in other studies, being in their mid-forties with disease duration of 13 years and with a similar prevalence of pneumothorax and angiomyolipoma [25]. Patients had moderate air-flow obstruction and impairment of gas transfer. Initial power calculations suggested we would require 20 patients per group to have $80 \%$ power to detect a $50 \%$ reduction in $\triangle \mathrm{FEV} 1$. Performing studies in rare diseases is difficult as patients need to be from a large area to deliver adequate study power, and only one randomised study of LAM has been published to date. Frequent pneumothoraces also limit recruitment of patients with LAM. Our recruitment criteria were broad and we linked study visits with patients' medical care where possible to facilitate participation in the study. Performing the study in a single centre ensures that procedures are standardised but the need for patients to travel limited recruitment to some extent. A more significant issue was that mTOR inhibitor therapy

TABLE 2 Sensitivity analysis comparing rate of decline in forced expiratory volume in $1 \mathrm{~s}$ $(\triangle \mathrm{FEV} 1)$ in each group for varying durations of treatment

\begin{tabular}{|c|c|c|c|c|c|}
\hline Duration of treatment & Treatment & Patients & $\begin{array}{c}\Delta \mathrm{FEV} 1 \\
\mathrm{~mL} \cdot \text { year }^{-1}\end{array}$ & Difference & $\mathrm{p}$-value \\
\hline Any (ITT analysis) & $\begin{array}{c}\text { Doxycycline } \\
\text { Placebo }\end{array}$ & $\begin{array}{l}12 \\
11\end{array}$ & $\begin{array}{r}-122.8 \pm 246 \\
-90.3+154\end{array}$ & $-32.5(-213-147.8)$ & 0.35 \\
\hline$>6$ months & $\begin{array}{l}\text { Doxycycline } \\
\text { Placebo }\end{array}$ & $\begin{array}{c}12 \\
9\end{array}$ & $\begin{array}{r}-122.8 \pm 246 \\
-61.5 \pm 112\end{array}$ & $-61.3(-247-124)$ & 0.25 \\
\hline$>12$ months & $\begin{array}{l}\text { Doxycycline } \\
\text { Placebo }\end{array}$ & $\begin{array}{l}9 \\
8\end{array}$ & $\begin{array}{l}-34.4 \pm 81 \\
-26.7 \pm 42\end{array}$ & $-7.7(-76-60)$ & 0.41 \\
\hline$>18$ months & $\begin{array}{l}\text { Doxycycline } \\
\text { Placebo }\end{array}$ & $\begin{array}{l}8 \\
7\end{array}$ & $\begin{array}{l}-33.5 \pm 86 \\
-39.6 \pm 24\end{array}$ & $6.1(-67-79)$ & 0.43 \\
\hline 24 months & $\begin{array}{l}\text { Doxycycline } \\
\text { Placebo }\end{array}$ & $\begin{array}{l}8 \\
7\end{array}$ & $\begin{array}{l}-33.5 \pm 86 \\
-39.6 \pm 24\end{array}$ & $6.1(-67-79)$ & 0.43 \\
\hline
\end{tabular}

Data are presented as $\mathrm{n}$, mean $\pm \mathrm{SD}$, or mean $(95 \% \mathrm{CI})$, unless otherwise stated. ITT: intention-to-treat. 


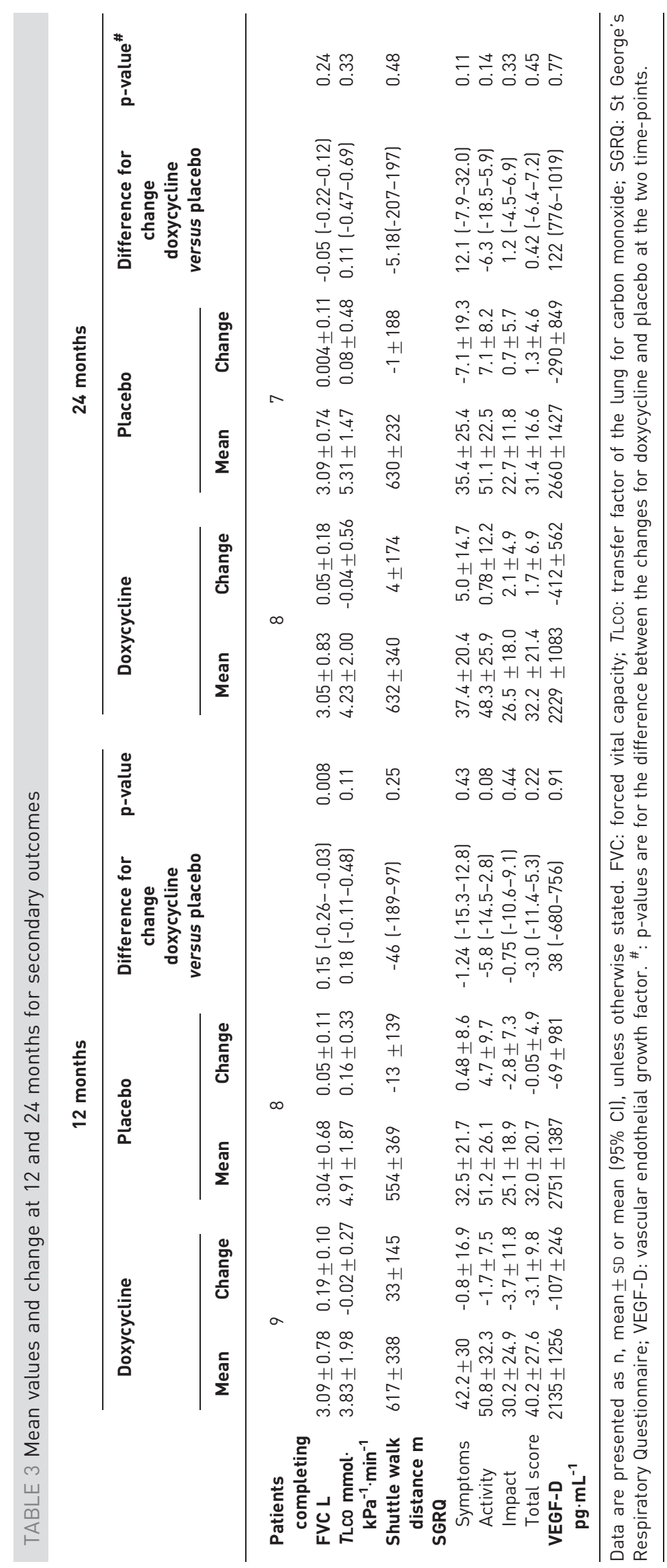


TABLE 4 Adverse events during the study

\begin{tabular}{|c|c|c|c|c|c|}
\hline \multirow[t]{2}{*}{ Category } & \multirow[t]{2}{*}{ All patients } & \multicolumn{2}{|c|}{ Doxycycline grade } & \multicolumn{2}{|c|}{ Placebo grade } \\
\hline & & $1-2$ & $3-4$ & $1-2$ & $3-4$ \\
\hline \multicolumn{6}{|l|}{ Auditory } \\
\hline Tinnitus $^{\#}$ & 1 & 1 & & & \\
\hline \multicolumn{6}{|l|}{ Dermatology } \\
\hline Dry skin & 2 & 1 & & 1 & \\
\hline Flushing & 3 & 2 & & 1 & \\
\hline Photosensitive rash & 2 & 1 & & 1 & \\
\hline Bruising & 1 & 1 & & & \\
\hline \multicolumn{6}{|l|}{ Gastrointestinal } \\
\hline Diarrhoea & 1 & 1 & & & \\
\hline Dyspepsia & 4 & 4 & & & \\
\hline \multicolumn{6}{|l|}{ Infection } \\
\hline Upper aerodigestive & 16 & 8 & 2 & 6 & \\
\hline \multicolumn{6}{|l|}{ Lymphatics } \\
\hline Chyle leak (chyloptysis) & 1 & & & 1 & \\
\hline \multicolumn{6}{|l|}{ Neurological } \\
\hline Neuropathy (motor) & 1 & 1 & & & \\
\hline Carpal tunnel & 1 & 1 & & & \\
\hline Seizure $^{+}$ & 1 & 1 & & & \\
\hline \multicolumn{6}{|l|}{ Pain } \\
\hline Headache & 3 & 1 & & 2 & \\
\hline Musculoskeletal & 1 & 1 & & & \\
\hline \multicolumn{6}{|l|}{ Respiratory } \\
\hline Dyspnoea & 2 & 1 & & 1 & \\
\hline FEV1 (fall) & 1 & 1 & & & \\
\hline Pneumothorax & 4 & 1 & & 2 & 1 \\
\hline Bronchospasm & 1 & 1 & & & \\
\hline \multicolumn{6}{|l|}{ Malignancy } \\
\hline Meningioma & 1 & 1 & & & \\
\hline Total & 45 & 29 & 2 & 15 & 1 \\
\hline
\end{tabular}

Data are presented as $\mathrm{n}$. Adverse events categorised by Common Terminology Criteria for Adverse Events version 3.0 [24]. No grade 5 events were observed. FEV1: forced expiratory volume in $1 \mathrm{~s}$. " : patient's had tinnitus prior to study, symptoms persisted on stopping drug; ": all patients were approaching the menopause; ${ }^{+}$: in a patient with meningioma.

became more widely available during the recruitment period and the enlarging evidence base for this therapy limited our ability to recruit patients with progressive disease to a placebo controlled study $[6,26]$. The study was designed to guard against patients with rapidly declining lung function receiving placebo for 2 years by incorporating stopping criteria for patients with a fall in FEV1 $>300 \mathrm{~mL}$ as a predetermined secondary endpoint. Two patients left the study early to receive sirolimus, one after a $>300 \mathrm{~mL}$ fall in FEV1.

In the intention-to-treat analysis the mean $\triangle \mathrm{FEV} 1$ was $-123 \mathrm{~mL} \cdot \mathrm{year}^{-1}$ in the doxycycline group and -90 $\mathrm{mL} \cdot$ year $^{-1}$ in the placebo group, values in keeping with previous reports. Patients who withdrew early had more rapid decline in lung function. The relatively low $\triangle \mathrm{FEV} 1$ in patients completing the study, $-35 \mathrm{~mL} \cdot$ year $^{-1}$, reflects the fact that patients with more aggressive disease were already receiving sirolimus or were opting for it. In addition, one third of our study population were post-menopausal when the decline in FEV1 is slower, which may make it more difficult to show a treatment effect. [2, 3] The 95\% CI for $\Delta$ FEV1 in the patients completing the study were tighter than in the intention-to-treat analysis, despite the smaller numbers, reflecting the fact that more measurements were made per patient over a longer period, and these patients had more stable disease. Nevertheless there was little difference in mean (95\% CI) $\Delta$ FEV1 between the two groups $\left(6.1 \mathrm{~mL} \cdot\right.$ year $\left.^{-1}(-67-79)\right)$. A limitation of the study was, due to chance, that patients randomised to doxycycline had slightly lower lung function than those receiving placebo. Although there is no definitive data on whether lung function varies with disease severity in LAM, it is possible that those with more severe disease may decline more rapidly although the converse is true in patients with $\alpha_{1}$-antitrypsin deficiency [27].

Two reports have described the effect of doxycycline on lung function. In a single patient with severe disease, lung function and oxygenation improved after doxycycline treatment although this may have been due treatment of a co-existing infection [17]. In a series of 38 patients all treated with doxycycline for 

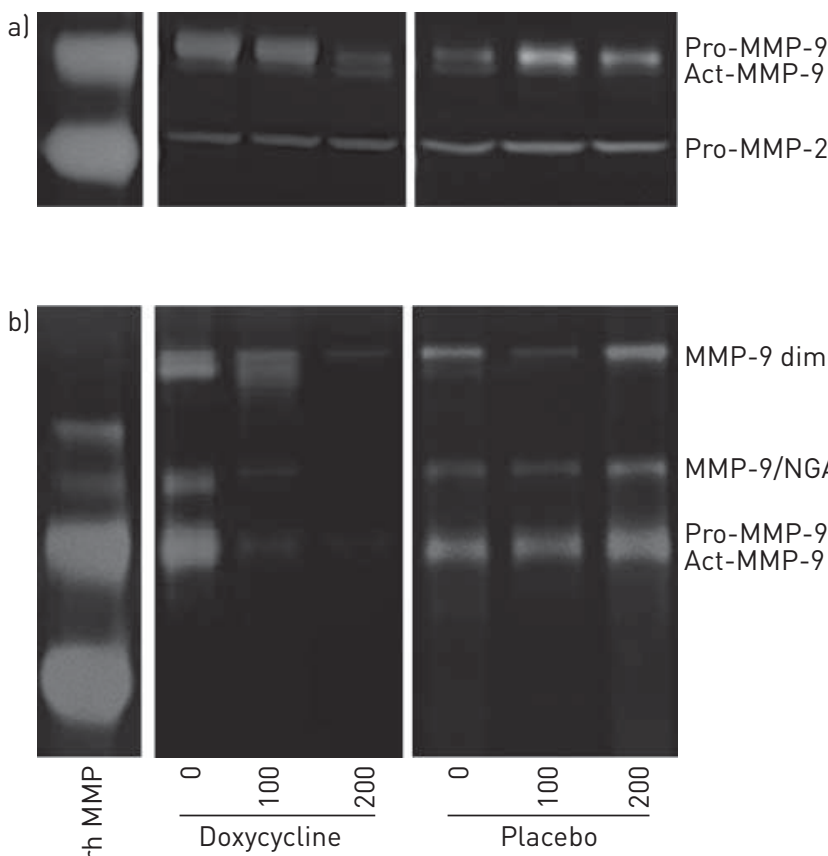

MMP-9 dimer

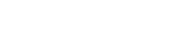

$\sum_{\substack{c \\ L}}^{n}$

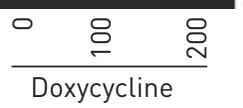

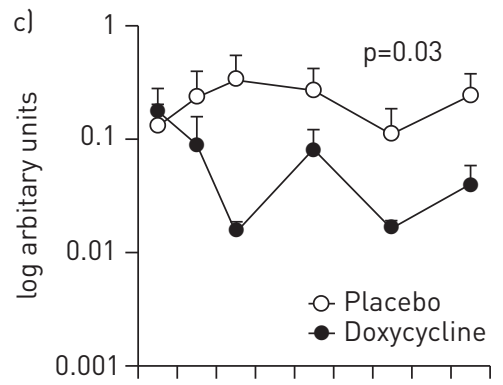

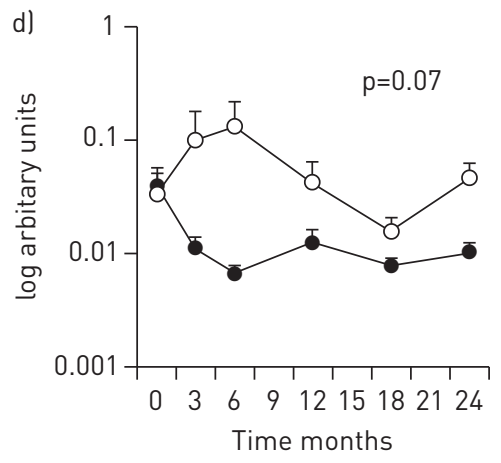

FIGURE 5 Gelatin zymograms of a) serum and b) urine from representative patients treated with doxycycline or placebo to detect matrix metalloproteinases (MMP)-2 and MMP-9. MMP species are visible as white areas of degraded gelatin. rh MMP: recombinant human MMP protein standard. NGAL: neutrophil gelatinase associated lipocalin. The mean \pm SE urinary c) total MMP-9 and d) active MMP-9 throughout the 2-year study is also shown. The p-values values are for overall differences between treatment groups.

12 months, PIMENTA et al. [19] observed that some patients remained stable on treatment and these tended to be those with better lung function. A relatively benign clinical course in some patients with LAM is well documented, and without a control group the significance of these findings is difficult to assess [28]. MMP2 and -9 are increased in LAM lung tissue [14] in cells derived from TSC knock-out animals [29] and in the serum and urine of patients with LAM [11], making them attractive candidates for causing the accelerated extra-cellular matrix destruction and cyst formation observed [13, 14, 30]. All the MMP measurements, but particularly those from urine, showed considerable intra- and inter-subject variation that was not obviously related to infection. Doxycycline treatment was associated with suppression of urinary MMP-9 but not serum MMP-9. Our failure to find a reduction in serum MMP-9 contrasts with the 5\% reduction seen by PIMENTA et. al. [19] and although this may reflect our smaller numbers and intra-subject variability, there must be doubt as to whether a change of $5 \%$ would result in clinical benefit. The lack of efficacy of doxycycline in preventing decline in lung function raises questions about the role of MMPs in lung destruction in LAM. However, the metalloproteinase system is complex with activating proteases and inhibitors interacting to regulate overall MMP activity both spatially and temporally. Further studies are required to determine whether MMPs are central to lung destruction in LAM and if more potent or selective targeting of individual proteases or their substrates could reduce lung destruction.

In summary, we found that treatment with doxycycline for 2 years had no effect on the decline in lung function in patients with LAM. It is common for patients with a rare disease to take off-label therapies on the basis of a biologically plausible mechanism of action but our findings provide no support to justify using doxycycline to treat LAM.

Specific targeting of lung destruction in LAM and other chronic lung diseases needs better understanding of the pathological mechanisms involved.

\section{Acknowledgements}

We are grateful to the participants taken from the lung function department at the Queens Medical Centre, Nottingham, UK, to Tim Harrison and Richard Hubbard (Division of Respiratory Medicine, University of Nottingham, Nottingham, UK) for their advice on the study design, and to Brendan Mallia-Milanes (Division of Therapeutics and Molecular Medicine, University of Nottingham, Nottingham, UK), Janet Bee and Sharon Fuller (Nottingham University Hospitals NHS Trust, Nottingham, UK) for their assistance with the administration of the study. 


\section{References}

Johnson SR. Lymphangioleiomyomatosis. Eur Respir J 2006; 27: 1056-1065.

2 Johnson SR, Tattersfield AE. Decline in lung function in lymphangioleiomyomatosis: relation to menopause and progesterone treatment. Am J Respir Crit Care Med 1999; 160: 628-633.

3 Taveira-DaSilva AM, Stylianou MP, Hedin CJ, et al. Decline in lung function in patients with lymphangioleiomyomatosis treated with or without progesterone. Chest 2004; 126: 1867-1874.

4 McCormack FX, Inoue Y, Moss J, et al. Efficacy and safety of sirolimus in lymphangioleiomyomatosis. N Engl J Med 2011; 364: 1595-606.

5 Henske EP, McCormack FX. Lymphangioleiomyomatosis a wolf in sheep's clothing. J Clin Inves 2012; 122 : 3807-3816.

6 Bissler JJ, McCormack FX, Young LR, et al. Sirolimus for angiomyolipoma in tuberous sclerosis complex or lymphangioleiomyomatosis. N Engl J Med 2008; 358: 140-151.

7 Davies DM, de Vries PJ, Johnson SR, et al. Sirolimus therapy for angiomyolipoma in tuberous sclerosis and sporadic lymphangioleiomyomatosis: a phase 2 trial. Clin Cancer Res 2011; 17: 4071-4081.

8 Juvet SC, McCormack FX, Kwiatkowski DJ, et al. Molecular pathogenesis of lymphangioleiomyomatosis: lessons learned from orphans. Am J Respir Cell Mol Biol 2007; 36: 398-408.

9 Chilosi M, Pea M, Martignoni G, et al. Cathepsin-k expression in pulmonary lymphangioleiomyomatosis. Mod Pathol 2009; 22: 161-166.

10 Greenlee KJ, Werb Z, Kheradmand F. Matrix metalloproteinases in lung: multiple, multifarious, and multifaceted. Physiol Rev 2007; 87: 69-98.

11 Odajima N, Betsuyaku T, Nasuhara Y, et al. Matrix metalloproteinases in blood from patients with LAM. Respir Med 2009; 103: 124-129.

12 Chang W, Cane J, Blakey J, et al. Clinical utility of diagnostic guidelines and putative biomarkers in lymphangioleiomyomatosis. Respir Res 2012; 13: 34.

13 Hayashi T, Fleming MV, Stetler-Stevenson WG, et al. Immunohistochemical study of matrix metalloproteinases (MMPs) and their tissue inhibitors (TIMPs) in pulmonary lymphangioleiomyomatosis (LAM). Hum Pathol 1997; 28: 1071-1078.

14 Matsui K, Takeda K, Yu ZX, et al. Role for activation of matrix metalloproteinases in the pathogenesis of pulmonary lymphangioleiomyomatosis. Arch Pathol Lab Med 2000; 124: 267-275.

15 Golub LM, Lee HM, Ryan ME, et al. Tetracyclines inhibit connective tissue breakdown by multiple nonantimicrobial mechanisms. Adv Dent Res 1998; 12: 12-26.

16 Bendeck MP, Conte M, Zhang M, et al. Doxycycline modulates smooth muscle cell growth, migration, and matrix remodeling after arterial injury. Am I Pathol 2002; 160: 1089-1095.

17 Moses MA, Harper J, Folkman J. Doxycycline treatment for lymphangioleiomyomatosis with urinary monitoring for MMPs. N Engl J Med 2006; 354: 2621-2622.

18 Pimenta SP, Baldi BG, Acencio MMP, et al. Doxiciclina em pacientes com linfangioleiomiomatose: segurança e eficácia no bloqueio de metaloproteinases [Doxycycline use in patients with lymphangioleiomyomatosis: safety and efficacy in metalloproteinase blockade]. J Bras Pneumol 2011; 37: 424-430.

19 Pimenta SP, Baldi BG, Kairalla RA, et al. Doxiciclina em pacientes com linfangioleiomiomatose: biomarcadores e resposta funcional pulmonar [Doxycycline use in patients with lymphangioleiomyomatosis: biomarkers and pulmonary function response]. J Bras Pneumol 2013; 39: 5-15.

20 Johnson SR, Cordier JF, Lazor R, et al. European Respiratory Society guidelines for the diagnosis and management of lymphangioleiomyomatosis. Eur Respir J 2010; 35: 14-26.

21 Bush A, Cramer D. Guidelines for the measurement of respiratory function. Respir Med 1994; 88: 798.

22 Lowrey GE, Henderson N, Blakey JD, et al. MMP-9 protein level does not reflect overall MMP activity in the airways of patients with COPD. Respir Med 2008; 102: 845-851.

23 Young LR, VanDyke R, Gulleman PM, et al. Serum vascular endothelial growth factor-D prospectively distinguishes lymphangioleiomyomatosis from other diseases. Chest 2010; 138: 674-681.

24 DCTD, NCI, NIH, DHHS. National Cancer Institute. Common Terminology Criteria for Adverse Events v3.0 (CTCAE). http://ctep.cancer.gov/protocolDevelopment/electronic_applications/docs/ctcaev3.pdf\#search Date last accessed November 11, 2013. Date last updated October 30, 2006.

25 Ryu JH, Moss J, Beck GJ, et al. The NHLBI lymphangioleiomyomatosis registry: characteristics of 230 patients at enrollment. Am J Respir Crit Care Med 2006; 173: 105-111.

26 Davies DM, Johnson SR, Tattersfield AE, et al. Sirolimus therapy in tuberous sclerosis or sporadic lymphangioleiomyomatosis. N Engl J Med 2008; 358: 200-203.

27 The Alpha-1-Antitrypsin Deficiency Registry Study Group. Survival and FEV1 decline in individuals with severe deficiency of $\alpha_{1}$-antitrypsin Am J Respir Crit Care Med 1998; 158: 49-59.

28 Johnson SR, Whale CI, Hubbard RB, et al. Survival and disease progression in UK patients with lymphangioleiomyomatosis. Thorax 2004; 59: 800-803.

29 Lee PS, Tsang SW, Moses MA, et al. Rapamycin-insensitive up-regulation of MMP2 and other genes in tuberous sclerosis complex 2-deficient lymphangioleiomyomatosis-like cells. Am J Respir Cell Mol Biol 2009; 42: 227-234.

30 Ferri N, Carragher NO, Raines EW. Role of discoidin domain receptors 1 and 2 in human smooth muscle cellmediated collagen remodeling: potential implications in atherosclerosis and lymphangioleiomyomatosis. Am J Pathol 2004; 164: 1575-1585. 